\title{
Analyzing the $\delta$ Sco binary in anticipation of a disk-star collision
}

\author{
Ashley Ames ${ }^{1}$, Christopher Tycner ${ }^{1}$ and Robert Zavala ${ }^{2}$ \\ ${ }^{1}$ Dept. of Physics, Central Michigan University \\ ${ }^{2}$ USNO, Flagstaff Station
}

\begin{abstract}
A current investigation is underway into the possible collision between a circumstellar disk and the secondary star in the $\delta$ Scorpii binary system. $\delta$ Scorpii is a prime candidate for a disk-star collision since the primary star has a circumstellar disk and the secondary star has a highly elliptical orbit with a period of approximately 10.5 years making the periastron passage very close to the primary star. The Navy Prototype Optical Interferometer (NPOI) was used to spatially resolve the two stars as well as the cirumstellar disk around the primary. A revised orbit with new orbital parameters has been calculated using observations obtained between 2005 and 2010. Our results indicate periastron passage will occur on 2011-07-03.
\end{abstract}

Keywords. instrumentation: high angular resolution, astrometry, binaries: general

\section{Introduction}

$\delta$ Scorpii (HD143275, HR5953, FK5 0594) is a well known binary system with a highly eccentric orbit and a period of 10.5 years. The primary star is classified as a Be star with a gaseous circumstellar disk and the secondary is a B2-type star (Tango et al. 2009). The goal for this project is to refine the orbit of the secondary with respect to the primary and test for the possibility of a disk-star collision. The $\delta$ Sco binary system was observed with the NPOI for a total of 108 nights. Data on two nights were obtained in July 2000 and the rest were obtained from June 2005 to August 2010.

\section{Experimental Data}

All the raw observational data, based on 108 nights, have been reduced using a standard NPOI reduction pipeline. The astrometric information is extracted from the reduced data giving the position of the secondary with respect to the primary. The NPOI observations were compared to orbits based on parameters from Mason et al. (2009), Tango et al. (2009), and Miroshnichenko et al. (2001). The new astrometric data, combined with radial velocities from Miroshnichenko et al. (2001), were used to calculate a refined orbit (shown in Figure 1) with the revised binary parameters listed in Table 1. Since the instrument used has a wide range of baselines, the resulting narrow-angle astrometry has high precision. In combination with extensive orbital coverage, including observations close to the previous periastron passage reults in the best orbital parameters to date.

\section{Summary}

The binary orbit has been refined using astrometric data obtained from NPOI and radial velocities from Miroshnichenko et al. (2001). The orbit that is obtained gives a better fit to the data than previous findings. The next periastron passage date has been 
Table 1. The orbital elements for $\delta$ Sco.

\begin{tabular}{|l|c|c|c|c|}
\hline Element & $\begin{array}{c}\text { Mason et al. } \\
(2009)\end{array}$ & $\begin{array}{c}\text { Miroshnichenko } \\
\text { et al. }(2001)\end{array}$ & $\begin{array}{c}\text { Tango et al. } \\
(2009)\end{array}$ & This Work \\
\hline Period(y) & $10.68 \pm 0.05$ & $10.58^{1}$ & $10.74 \pm 0.02$ & $10.81 \pm 0.002$ \\
Semimajor axis (mas) & $104 \pm 6$ & $107^{1}$ & $98.3 \pm 1.2$ & $99.0 \pm 0.05$ \\
inclination, i (deg) & $39 \pm 8$ & $38 \pm 5$ & $38 \pm 6$ & $30.3 \pm 0.24$ \\
Long. of asc. node, $\Omega(\mathrm{deg})$ & $153 \pm 9$ & $175^{1}$ & $175.2 \pm 0.6$ & $172.3 \pm 0.6$ \\
eccentricity, e & $0.94^{2}$ & $0.94 \pm 0.01$ & $0.9401 \pm 0.0002$ & $0.941 \pm 0.0007$ \\
Long. periastron, $\omega$ & $29 \pm 12$ & $-1 \pm 5$ & $1.9 \pm 0.1$ & $2.6 \pm 0.7$ \\
T (Epoch of & $J 2000.693^{2}$ & $J 2000.693 \pm$ & $J 2000.69389 \pm$ & $J 2000.6942 \pm$ \\
Periastron) & & 0.008 & 0.00007 & 0.0011 \\
\hline
\end{tabular}

Notes:

1 Parameter adopted from Hartkopf et al. (1996) solution.

2 Parameter adopted from Miroshnichenko et al. (2001) solution.

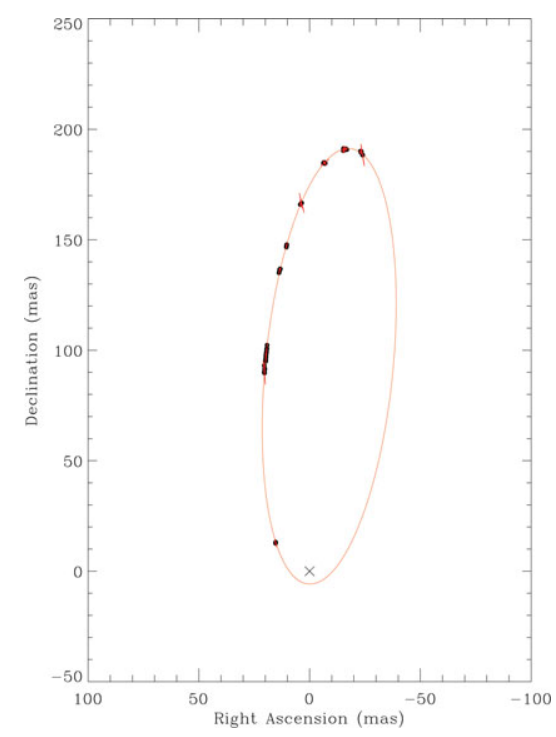

Figure 1. Binary orbit based on orbital parameters obtained from NPOI data. The black circles are the data points and the locations of the primary is marked with an X.

calculated to be 2011-07-02, $22 h \pm 1.1 d$. Our results indicate the periastron passage will occur 24 days later than the prediction of Tango et al. (2009).

\section{Acknowledgements}

The Navy Prototype Optical Interferometer is a joint project of the Naval Research Laboratory and the US Naval Observatory, in cooperation with Lowell Observatory, and is funded by the Office of Naval Research and the Oceanographer of the Navy. A.A. and C.T. would also like to thank the Physics Department at Central Michigan University for support.

\section{References}

Mason, B. D., Hartkopf, W. I., Gies, D. R., Henry, T. J. et al. 2009, AJ, 137, 3358

Hartkopf, W. I., Mason, B. D., \& McAlister, H. A. 1996, AJ, 111, 370

Miroshnichenko, A. S., Fabregat, J., Bjorkman, K. S., Knauth, D. C. et al. 2001, A\&A, 377, 485

Tango, W. J., Davis, J., Jacob, A. P., Mendez, A. et al. 2009, MNRAS, 396, 842 